\title{
OCCULT PRIMARY TRIPLE NEGATIVE BREAST CANCER IN AN ELDERLY PATIENT: CASE REPORT
}

Anna Carolina Pereira Jacome1, Ingrid Bernucci Neto', Patrícia Aguiar Bellini', Luciana Carvalho Horta1', Bruno Henrique Jacome Alvarenga ${ }^{1}$

${ }^{1}$ Hospital Felício Rocho - Belo Horizonte (MG), Brazil.

Introduction: Occult primary breast cancer is very rare, accounting for less than $1 \%$ of all cases of breast cancer, generally associated with a poor prognosis. It is defined as a clinically recognizable metastatic carcinoma derived from an undetectable primary breast tumor, with metastasis to the axillary and cervical lymph nodes. Clinical and radiological examinations represent the first steps in the diagnosis, followed by a histological and immunohistochemistry (IHC) analysis, as well as a multidisciplinary team evaluation and therapy - essential for diagnosis and treatment. The most common phenotype is a positive hormone-receptor adenocarcinoma for which there is no clear consensus about optimal management, however a standard approach is axillary lymph node (ALN) dissection. Ipsilateral mastectomy, neoadjuvant chemotherapy and radiotherapy are controversial but may be acceptable in selected cases. Case report: A 72-year-old woman with a history of colon adenocarcinoma surgically treated, presented with an axillary mass of rapid growth. Uponn physical examination, a $5 \mathrm{~cm}$ mass in the left axilla and a palpable ipsilateral supraclavicular lymph node (SCLN) were identified, without any evidence of a breast lesion. imaging analysis with bilateral mammography, ultrasonography and breast magnetic ressonance imaging showed suspicious axillary and SC adenopathy, both on the left side; no abnormal breast findings. She was submitted to core biopsy and IHC analysis, andan invasive triple negative metastatic breast cancer was diagnosed. The patient underwent neoadjuvant chemotherapy with cyclophosphamide / doxorubicin, evolving with disease progression, so the regimen was modified to carboplatin. There was no response to treatment, with persistent growing of the lesion. Neoadjuvant chemotherapy was interrupted and surgery was performed to resect the ALN and the SCLN. It was a difficult surgery due to the extension of the axillary mass, in conjunction with adherence to the subclavian vein. A histologic analysis confirmed the inicial diagnosis of metastatic breast cancer. Surgery was followed by radiotherapy, but disease progression was fast. She manifested a large axillary recurrence and progressed to death 4 months after the beginning of treatment. This case report describes how challeging occult breast cancer can be, specially when associated with an unusual presentation such as a triple negative phenotype and SC adenopathy. At first, the hypothesis of colon metastasis was proposed due to the poor reponse to chemotherapy. Despite being submited to the standard approach proposed and supported by literature, the aggressive and rapid progression to death represents an obvious need to discuss other treatment options for occult breast carcinoma with unusual presentations, such as negative hormone-receptor. 